\title{
Plasmid-mediated quinolone resistance in Salmonella from South Africa
}

Invasive infections with Salmonella species are usually treated with fluoroquinolones or extended-spectrum $\beta$-lactams. Quinolone resistance results in reduced susceptibility to fluoroquinolones, leaving health-care professionals with limited resources for treatment of salmonellosis. Several mechanisms contribute to the development of quinolone resistance in Salmonella and these include: mutations in the quinolone resistance-determining region (QRDR) of DNA gyrase ( $g y r A / g y r B)$ and/or topoisomerase IV (parC/parE) genes (Eaves et al., 2004); active efflux of antibiotic out of the bacterial cell (Baucheron et al., 2002); and plasmidmediated quinolone resistance encoded by $q n r$ genes $A, B$ and $S$ (Robicsek et al., 2006a). The qnr genes encode pentapeptide repeat proteins that block the action of quinolones on bacterial DNA gyrase and topoisomerase IV, resulting in low-level quinolone resistance (Tran et al., 2005a, b). The first qnr gene was isolated in 1998 from a Klebsiella pneumoniae isolate from Birmingham, Alabama, USA, and was named $q n r$ (later renamed $q n r A$ ) (Martinez-Martinez et al., 1998). qnr genes have been identified in various bacterial species worldwide (Robicsek et al., 2006a). More recently, a novel quinoloneresistance mechanism has been identified in K. pneumoniae which involves the modification of certain fluoroquinolones by a specific type of aminoglycoside acetyltransferase (Robicsek et al., 2006b). We report what we believe to be the first discovery of plasmid-mediated quinolone resistance in Salmonella from South Africa, which was identified in a clinical isolate of Salmonella enterica serovar Typhimurium.

A subset $(n=48)$ of invasive $S$.

Typhimurium isolates collected by the Enteric Diseases Reference Unit of the National Institute for Communicable Diseases in the year 2006, with MICs for nalidixic acid of $\geqslant 32 \mu \mathrm{g} \mathrm{ml}{ }^{-1}$, were investigated. Susceptibility testing to antimicrobial agents was determined by the Etest (AB BIODISK). The presence of extended-spectrum $\beta$-lactamase (ESBL) activity was investigated using disc diffusion screening methods, as described by the Clinical and Laboratory Standards Institute (CLSI, 2005). Isolates were screened for $q n r A$, qnrB and $q n r S$ genes. Individual PCRs were used to screen for each type of $q n r$ gene, using previously described methods (Jacoby et al., 2003, 2006; Hopkins et al., 2007). PCR-positive $q n r$ genes were sequenced. Isolates which were PCR-positive for a $q n r$ gene were further investigated by PCR and sequencing of the QRDR of $g y r A$, gyrB, parC and parE, using previously described methods (Eaves et al., 2004). DNA sequencing reactions were carried out using the BigDye Terminator Cycle Sequencing kit (Applied Biosystems) and DNA sequences were determined using the Applied Biosystems 3130 genetic analyser. Analysis of DNA sequences was performed using the DNASTAR Lasergene (version 8.0) software. For nalidixic acid and ciprofloxacin, agar dilution MIC testing was performed in the presence and absence of the efflux pump inhibitor Phe-Arg- $\beta$-naphthylamide (Baucheron et al., 2002). Agar dilution MIC testing was performed using methods as described by the Clinical and Laboratory Standards Institute (CLSI, 2005).

From all isolates tested, we identified a single isolate (nalidixic acid MIC $32 \mu \mathrm{g}$ $\mathrm{ml}^{-1}$; ciprofloxacin MIC $0.38 \mu \mathrm{g} \mathrm{ml}^{-1}$ ) which was PCR-positive for the $q n r B$ gene. This isolate was cultured from the blood of an HIV-seropositive 44-year-old female with a CD4 count of $31 \mu \mathrm{l}^{-1}$. The patient was hospitalized from a tuberculosis sanatorium with chronic diarrhoea. Stool culture did not yield an organism. The patient was treated with ciprofloxacin, $500 \mathrm{mg}$ 12-hourly for 14 days. She remains well on antiretroviral therapy 2 years later. The PCR-positive qnrB result was confirmed by nucleotide sequence analysis of the gene. Sequencing revealed the qnrB2 variant. Sequence analysis of the QRDR of
gyrA, gyrB, parC and parE for this isolate showed no mutations, therefore mutation in the QRDR of DNA gyrase and topoisomerase IV genes was excluded as a mechanism for quinolone resistance. Agar dilution MICs for nalidixic acid and ciprofloxacin showed no involvement in resistance of an active efflux pump, as there was no difference in MIC values following testing in the presence or absence of an efflux pump inhibitor. Agar dilution MIC testing confirmed the isolate's low-level nalidixic acid resistance (MIC $32 \mu \mathrm{g} \mathrm{ml}^{-1}$ ) and decreased susceptibility to ciprofloxacin (MIC $0.38 \mu \mathrm{g} \mathrm{ml}^{-1}$ ). These observed MIC values compared favourably with previously reported MIC values in association with qnrB (Chmelnitsky et al., 2008; GarciaFernandez et al., 2009; Gay et al., 2006). The low-level nalidixic acid resistance and decreased susceptibility to ciprofloxacin of our $S$. Typhimurium isolate was therefore most likely conferred by the QnrB protein. With regards resistance to other antimicrobial agents, our isolate also showed resistance to ampicillin, augmentin, trimethoprim, sulfamethoxazole, tetracycline, streptomycin and kanamycin, while showing susceptibility to chloramphenicol, ceftriaxone, ceftazidime and imipenem. No ESBL activity was detected.

Although the patient from whom our isolate was obtained recovered well after receiving ciprofloxacin therapy, isolates such as these that exhibit low-level quinolone resistance due to $q n r$ genes facilitate the selection of higher-level resistance mutants. $S$.

Typhimurium is the most common serotype isolated in South Africa (K. H. Keddy, unpublished results) and South Africa has a high burden of HIV, so the isolation of $S$. Typhimurium showing plasmid-mediated quinolone resistance from an immunocompromised patient illustrates the possible treatment challenges for these patients when drug interactions have to be taken into consideration. In these situations, 
carbapenems could be used to combat quinolone-resistant and ESBL-producing strains. Our study has shown that the $q n r B$ gene is a contributing factor for low-level quinolone resistance in $S$. Typhimurium. We believe that this is the first report of any kind identifying the presence of $q n r$ genes in South African Salmonella isolates.

\section{Acknowledgements}

This work was financially supported by the following South African institutions: the National Health Laboratory Service, the Medical Research Council and the National Research Foundation.

\section{Nevashan Govender, ${ }^{1}$ Anthony M. Smith, ${ }^{1,2}$ Alan S. Karstaedt ${ }^{2,3}$ and Karen H. Keddy ${ }^{1,2}$, for the Group for Enteric, Respiratory and Meningeal Disease Surveillance in South Africa (GERMS-SA) \\ ${ }^{1}$ Enteric Diseases Reference Unit, National Institute for Communicable Diseases, Johannesburg, South Africa \\ ${ }^{2}$ University of the Witwatersrand, Johannesburg, South Africa \\ ${ }^{3}$ Chris Hani Baragwanath Hospital, Johannesburg, South Africa}

Correspondence: Anthony M. Smith (anthonys@nicd.ac.za)
Baucheron, $\mathrm{S}_{\text {, }}$ Imberechts, $\mathrm{H}_{\text {, }}$ ChaslusDancla, E. \& Cloeckaert, A. (2002). The AcrB multidrug transporter plays a major role in high-level fluoroquinolone resistance in Salmonella enterica serovar Typhimurium phage type DT204. Microb Drug Resist 8, 281-289.

Chmelnitsky, I., Navon-Venezia, S., Strahilevitz, J. \& Carmeli, Y. (2008). Plasmidmediated qnrB2 and carbapenemase gene $b l a_{\mathrm{KPC}-2}$ carried on the same plasmid in carbapenem-resistant ciprofloxacin-susceptible Enterobacter cloacae isolates. Antimicrob Agents Chemother 52, 2962-2965.

CLSI (2005). Performance Standards for Antimicrobial Susceptibility Testing; Fifteenth Informational Supplement. CLSI document M100-S15. Wayne, PA: Clinical and Laboratory Standards Institute.

Eaves, D. J., Randall, L., Gray, D. T., Buckley, A., Woodward, M. J., White, A. P. \& Piddock, L. J. (2004). Prevalence of mutations within the quinolone resistance-determining region of gyrA, gyrB, parC, and parE and association with antibiotic resistance in quinolone-resistant Salmonella enterica. Antimicrob Agents Chemother 48, 4012-4015.

Garcia-Fernandez, A., Fortini, D., Veldman, K., Mevius, D. \& Carattoli, A. (2009).

Characterization of plasmids harbouring qnrS1, qnrB2 and qnrB19 genes in Salmonella.

J Antimicrob Chemother 63, 274-281.

Gay, K., Robicsek, A., Strahilevitz, J., Park, C. H., Jacoby, G., Barrett, T. J., Medalla, F., Chiller, T. M. \& Hooper, D. C. (2006). Plasmidmediated quinolone resistance in non-Typhi serotypes of Salmonella enterica. Clin Infect Dis 43, 297-304.
Hopkins, K. L, Wootton, L., Day, M. R. \& Threlfall, E. J. (2007). Plasmid-mediated quinolone resistance determinant qnrS1 found in Salmonella enterica strains isolated in the UK. J Antimicrob Chemother 59, 1071-1075.

Jacoby, G. A., Chow, N. \& Waites, K. B. (2003). Prevalence of plasmid-mediated quinolone resistance. Antimicrob Agents Chemother 47, 559-562.

Jacoby, G. A., Walsh, K. E., Mills, D. M., Walker, V. J., Oh, H., Robicsek, A. \& Hooper, D. C. (2006). qnrB, another plasmid-mediated gene for quinolone resistance. Antimicrob Agents Chemother 50, 1178-1182.

Martinez-Martinez, L., Pascual, A. \& Jacoby, G. A. (1998). Quinolone resistance from a transferable plasmid. Lancet 351, 797-799.

Robicsek, A., Jacoby, G. A. \& Hooper, D. C. (2006a). The worldwide emergence of plasmidmediated quinolone resistance. Lancet Infect Dis 6, 629-640.

Robicsek, A., Strahilevitz, J., Jacoby, G. A., Macielag, M., Abbanat, D., Park, C. H., Bush, K. \& Hooper, D. C. (2006b). Fluoroquinolonemodifying enzyme: a new adaptation of a common aminoglycoside acetyltransferase. Nat Med 12, 83-88.

Tran, J. H., Jacoby, G. A. \& Hooper, D. C. (2005a). Interaction of the plasmid-encoded quinolone resistance protein Qnr with Escherichia coli DNA gyrase. Antimicrob Agents Chemother 49, 118-125.

Tran, J. H., Jacoby, G. A. \& Hooper, D. C. (2005b). Interaction of the plasmid-encoded quinolone resistance protein QnrA with Escherichia coli topoisomerase IV. Antimicrob Agents Chemother 49, 3050-3052. 